\title{
Usefulness of Pure Laparoscopic Hepatectomy for Hepatocellular Carcinoma in a Severely Cirrhotic Patient
}

\author{
Isamu Hosokawa Hideyuki Yoshitomi Hiroaki Shimizu \\ Tsukasa Takayashiki Masaru Miyazaki \\ Department of General Surgery, Chiba University Graduate School of Medicine, Chiba, \\ Japan
}

\section{Key Words}

Pure laparoscopic hepatectomy · Hepatocellular carcinoma $\cdot$ Severely cirrhotic patient

\begin{abstract}
The number of patients undergoing laparoscopic hepatectomy has rapidly increased in recent years, and indications for this procedure are gradually expanding. Pure laparoscopic hepatectomy is reportedly useful in cases with severe liver cirrhosis. A 55-year-old woman under observation for liver cirrhosis was found to have hepatocellular carcinoma in liver segment III and was referred to our hospital for surgery. The tumor was located in the edge of liver segment III, where percutaneous ablation therapy was unsuitable. Since her hepatic functional reserve was poor, pure laparoscopic partial hepatectomy was performed. The postoperative course was favorable, with no ascites retention, edema or weight gain. The greatest advantage of pure laparoscopic hepatectomy for hepatocellular carcinoma with concomitant liver cirrhosis is that postoperative ascites retention is minimal, meaning that there is little risk of water-electrolyte imbalance associated with ascites retention or hypoproteinemia. This is believed to be because the abdominal incision is small and mobilization of the liver is minimized, reducing the destruction of the routes of collateral lymph flow and blood flow generated in patients with liver cirrhosis. Pure laparoscopic hepatectomy may be a treatment choice for patients with hepatocellular carcinoma and concomitant severe liver cirrhosis.

(c) 2013 S. Karger AG, Basel
\end{abstract}


Hosokawa et al.: Usefulness of Pure Laparoscopic Hepatectomy for Hepatocellular Carcinoma in a Severely Cirrhotic Patient

\section{Introduction}

Hepatocellular carcinoma (HCC) is the most common primary liver cancer with an increased incidence in patients with cirrhosis. Patients with cirrhosis have a high risk of morbidity and mortality when administered anesthesia and undergoing surgery. In particular, hepatic resection in patients with severe cirrhosis, even when the hepatectomy is quite limited, often leads to the development of refractory ascites, which in turn leads to fatal complications such as postoperative liver failure [1].

Laparoscopic hepatectomy is thought to be a less invasive procedure than conventional open hepatectomy [2]. Because of this, the number of cases of laparoscopic hepatectomy has rapidly increased in recent years, and the indications for this procedure are gradually expanding due to improvements in surgical instruments and techniques [1-5]. Accordingly, the usefulness of pure laparoscopic hepatectomy for patients with HCC and concomitant severe liver cirrhosis has been reported [1]. We recently experienced a case of HCC in a severely cirrhotic patient who was successfully treated with pure laparoscopic hepatectomy, and we herein present this case.

\section{Case Report}

A 55-year-old Japanese woman had been observed at a local hospital for 10 years for liver cirrhosis of unknown cause. A 20-mm tumor was found in liver segment III (S3), and she was referred to our hospital for surgery. Her laboratory data on admission were as follows: platelet count $7.8 \times 10^{4} / \mathrm{dl}$ (normal 13.0-36.0), total bilirubin $1.4 \mathrm{mg} / \mathrm{dl}$ (normal 0.2-1.1), albumin $3.3 \mathrm{~g} / \mathrm{dl}$ (normal 3.8-5.3), prothrombin time 88\% (normal 70-140), and ammonia $95 \mu \mathrm{g} / \mathrm{dl}$ (normal 30-86). Her hepatic functional reserve was as follows: indocyanine green retention rate at $15 \mathrm{~min} 72.7 \%$ (normal $0-10$ ) and galactose tolerance test 40 min (normal $\leq 20 \mathrm{~min}$ ). Abdominal enhanced computed tomography (CT) showed a 20-mm tumor that was heterogeneously enhanced during the early phase (fig. 1a) and washed out during the late phase (fig. 1b) on the surface of S3. Gd-EOB-DTPA-enhanced magnetic resonance imaging showed a hypointense region in S3 as seen on CT in the hepatocyte phase (fig. 1c). Although the patient's hepatic functional reserve was poor, the tumor was located in the edge of the liver and we thus considered percutaneous ablation therapy, such as radiofrequency ablation, to be unsuitable because of concern about hemorrhage, tumor dissemination and injury to adjacent organs. Therefore, we performed pure laparoscopic partial hepatectomy of S3.

The patient was placed supine, in the head-up tilt position, with the primary surgeon on the right side of the patient. Five ports were placed including 12-mm ports for the camera and intraoperative ultrasonography (fig. 2a). Intra-abdominal pressure was maintained at $10-12 \mathrm{~mm} \mathrm{Hg}$. Operative findings revealed that a $20-\mathrm{mm}$ white tumor protruding outside the liver was present in S3 (fig. 2b). A Cavitron ultrasonic surgical aspirator and salineenhanced coagulation were used to perform partial hepatectomy of S3 with a margin of $1 \mathrm{~cm}$ without vascular inflow occlusion. Operative time was $170 \mathrm{~min}$ and operative blood loss was $350 \mathrm{ml}$. A 20-mm, comparatively well-demarcated, septate white tumor was present in the resected specimen (fig. 2c). Histopathologically, the lesion was identified as moderately differentiated HCC without vascular invasion, and the surgical margin was negative for cancer (fig. 2d).

The patient's postoperative course was extremely uneventful. Her drain discharge was serous, with a volume of around $100 \mathrm{ml}$ until postoperative day 3; the drainage decreased to 
Hosokawa et al.: Usefulness of Pure Laparoscopic Hepatectomy for Hepatocellular Carcinoma in a Severely Cirrhotic Patient

$30 \mathrm{ml}$ on day 4. The drain was therefore removed on day 5 . There was no subsequent ascites retention, edema or weight gain. Her serum liver enzyme levels were transiently elevated postoperatively, but rapidly decreased again. There was no rise in total bilirubin or ammonia levels postoperatively. The patient was discharged on postoperative day 8 with no other complications (fig. 3). As of the time of this writing (10 months postoperatively), the patient is doing well clinically with no signs of recurrence.

\section{Discussion}

Indications for hepatectomy to treat HCC must be determined based on the balance between tumor conditions and hepatic functional reserve [6-8]. Selection of the most appropriate surgical procedure according to tumor progression within tolerable limits for hepatectomy is important [6]. However, patients with severe liver cirrhosis who undergo hepatectomy often develop postoperative liver failure, even when the hepatectomy is quite limited [2, 3].

The greatest advantage of laparoscopic hepatectomy for HCC with concomitant liver cirrhosis is that there is very little postoperative ascites retention [1-5], which leads to lower risk of water-electrolyte imbalance and hypoproteinemia [1]. This is believed to be because the abdominal incision is small and mobilization of the liver is greatly reduced, minimizing the destruction of the routes of collateral lymph flow and blood flow generated in patients with liver cirrhosis [1-5]. Pure laparoscopic hepatectomy in particular involves the least amount of abdominal wall destruction and is therefore highly effective. Other advantages include not only the common benefits of laparoscopic surgery, such as mild postoperative pain and the ability to achieve early ambulation and oral food intake [1,4], but also minimization of the mesenchymal injury caused by direct manipulation of the liver $[1,5]$. Furthermore, since there is little insensible perspiration and the detached area is small, minimal fluid replacement is required during the surgery and the risk of edema is lower [4, 5]. Pneumoperitoneal pressure also exerts a hemostatic effect, reducing the need to use the Pringle maneuver or other means of inflow occlusion during hepatic resection and minimizing ischemia-reperfusion injury in the liver [2].

Fewer postoperative adhesions are seen compared with conventional open hepatectomy, and for tumors on the liver surface, the procedure carries a lower risk of peritoneal dissemination than does radiofrequency ablation, making it ideal as a bridging therapy for liver transplantation $[3,4]$. The histological type and major vascular invasion can also be evaluated from the resected specimen, making this approach useful for assessing whether the patient can be safely referred for transplantation $[2,3,9,10]$.

Although indications for pure laparoscopic hepatectomy are currently limited by tumor conditions such as localization and size, conventional open hepatectomy need not be chosen on the grounds of poor hepatic functional reserve. In the future, if the patients can be appropriately selected, the indications for pure laparoscopic hepatectomy for HCC may be expanded to include severely cirrhotic patients.

In conclusion, pure laparoscopic hepatectomy for HCC with severe liver cirrhosis is useful in terms of minimal postoperative ascites, which leads to lower risks of developing water-electrolyte imbalance and hypoproteinemia. Pure laparoscopic hepatectomy may be a treatment choice for patients with HCC and concomitant severe liver cirrhosis. 
Hosokawa et al.: Usefulness of Pure Laparoscopic Hepatectomy for Hepatocellular Carcinoma in a Severely Cirrhotic Patient

\section{Disclosure Statement}

The authors declare that they have no conflicts of interest.

\section{References}

1 Morise Z, Sugioka A, Kawanabe N, Umemoto S, Nagata H, Ohshima H, Kawase J, Arakawa S, Yoshida R: Pure laparoscopic hepatectomy for hepatocellular carcinoma patients with severe liver cirrhosis. Asian J Endosc Surg 2011;4:143-146.

-2 Belli G, Fantini C, D’Agostino A, Cioffi L, Langella S, Russolillo N, Belli A: Laparoscopic versus open liver resection for hepatocellular carcinoma in patients with histologically proven cirrhosis: short- and middleterm results. Surg Endosc 2007;21:2004-2011.

-3 Cherqui D, Laurent A, Tayar C, Chang S, Van Nhieu JT, Loriau J, Karoui M, Duvoux C, Dhumeaux D, Fagniez PL: Laparoscopic liver resection for peripheral hepatocellular carcinoma in patients with chronic liver disease. Ann Surg 2006;243:499-506.

4 Kaneko H, Tsuchiya M, Otsuka Y, Yajima S, Minagawa T, Watanabe M, Tamura A: Laparoscopic hepatectomy for hepatocellular carcinoma in cirrhotic patients. J Hepatobiliary Pancreat Surg 2009;16:433-438.

-5 Laurent A, Cherqui D, Lesurtel M, Brunetti F, Tayar C, Fagniez PL: Laparoscopic liver resection for subcapsular hepatocellular carcinoma complicating chronic liver disease. Arch Surg 2003;138:763-769.

-6 Imamura H, Sano K, Sugawara Y, Kokudo N, Makuuchi M: Assessment of hepatic reserve for indication of hepatic resection: decision tree incorporating indocyanine green test. J Hepatobiliary Pancreat Surg 2005;12:16-22.

7 Mansour A, Watson W, Shayani V, Pichleman J: Abdominal operations in patients with cirrhosis: still a major surgical challenge. Surgery 1997;122:730-736.

-8 Belghiti J, Hiramatsu K, Benoist S, Massault PP, Sauvanet A, Farges O: Seven hundred forty-seven hepatectomies in the 1990s: an update to evaluate the actual risk of liver resection. J Am Coll Surg 2000;191:38-46.

-9 Buell JF, Thomas MT, Rudich S, Marvin M, Nagubandi R, Ravindra KV, Brock G, McMasters KM: Experience with more than 500 minimally invasive hepatic procedures. Ann Surg 2008;248:475-486.

-10 Laurent A, Tayar C, Andreoletti M, Lauzet JY, Merle JC, Cherqui D: Laparoscopic liver resection facilitates salvage liver transplantation for hepatocellular carcinoma. J Hepatobiliary Pancreat Surg 2009;16:310-314.
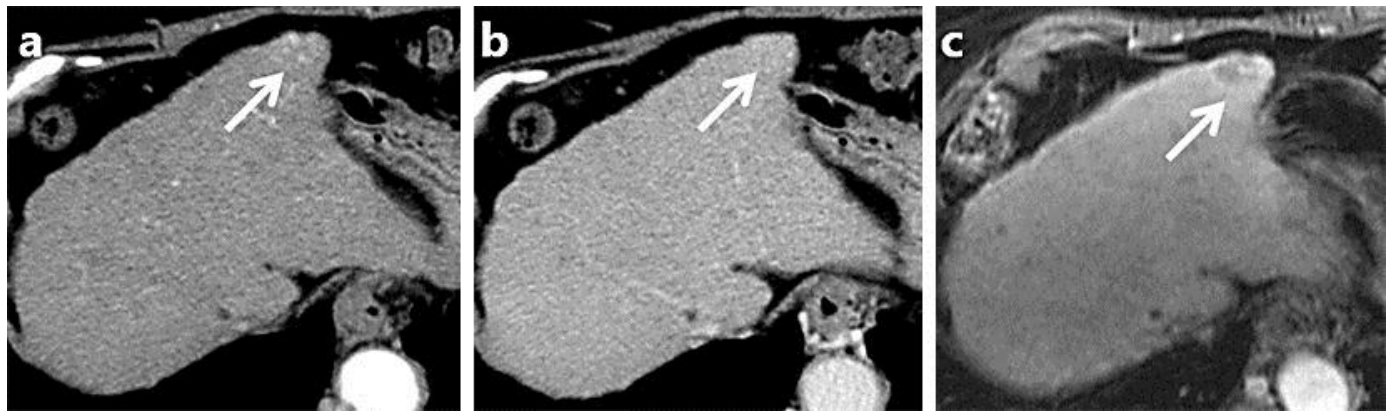

Fig. 1. Preoperative imaging findings. Abdominal enhanced CT showed a 20-mm tumor that was heterogeneously enhanced during the early phase (a) and washed out during the late phase (b) in the edge of S3 (arrows). Gd-EOB-DTPA-enhanced magnetic resonance imaging showed a hypointense region in S3 as seen on CT in the hepatocyte phase (c) (arrow). 


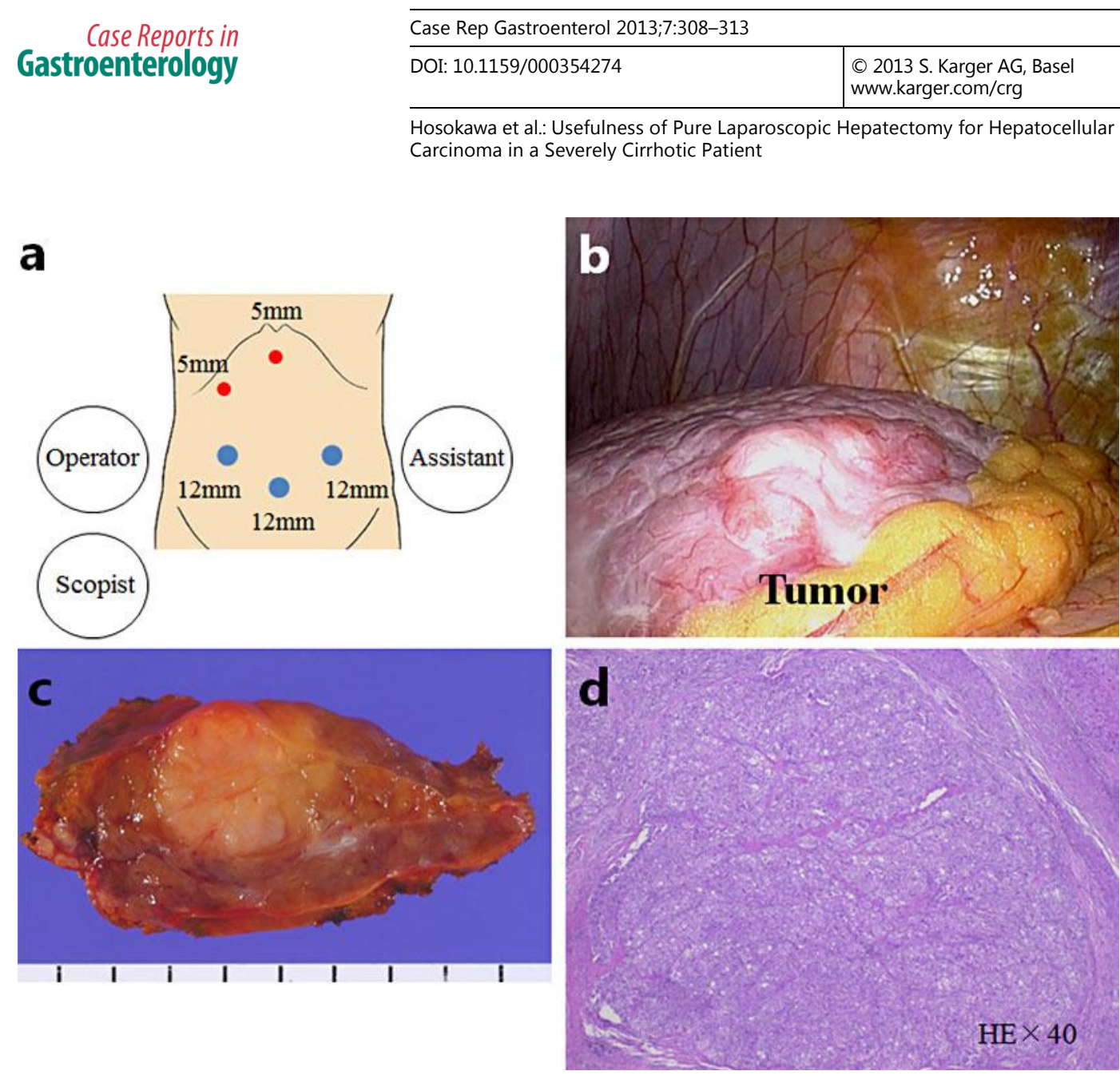

Fig. 2. Operative findings and the resected specimen. a 5 ports were placed including 12 -mm ports for the camera and intraoperative ultrasonography. $\mathbf{b}$ The liver was very cirrhotic with an irregular surface. A 20-mm white tumor protruding outside the liver was present in S3. c Gross examination of the resected specimen revealed that a $20-\mathrm{mm}$, comparatively well-demarcated, septate white tumor was present. d Histopathologic examination of the resected specimen revealed that the lesion was moderately differentiated HCC without vascular invasion, and the surgical margin was negative for cancer. 
Hosokawa et al.: Usefulness of Pure Laparoscopic Hepatectomy for Hepatocellular

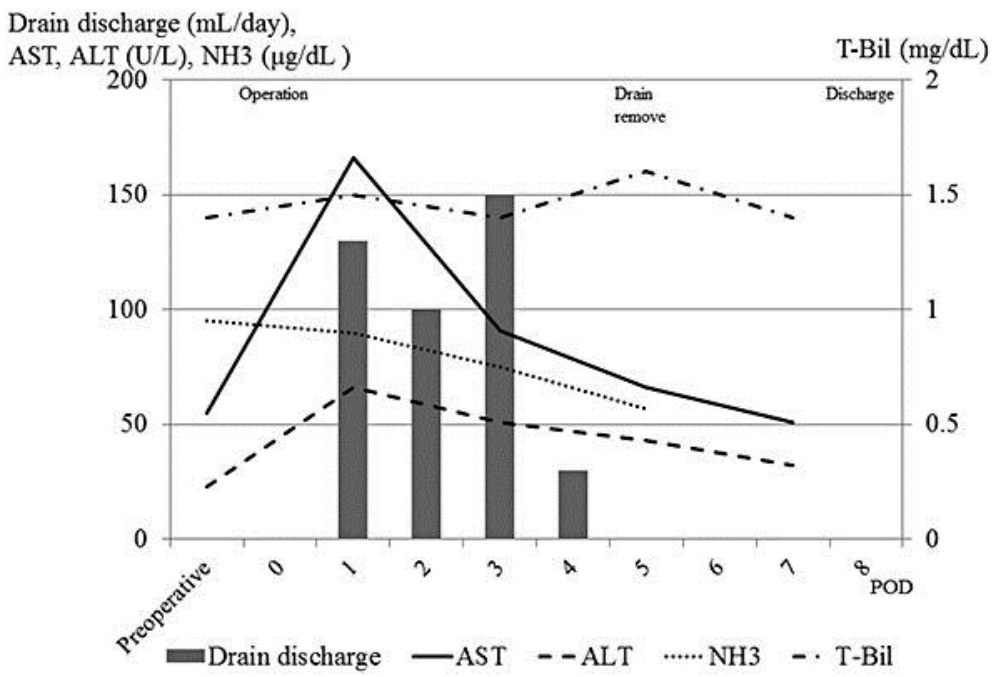

Fig. 3. Postoperative course. The drain discharge was serous, with a volume of around $100 \mathrm{ml}$ until postoperative day 3; the drainage decreased to $30 \mathrm{ml}$ on day 4 . The drain was therefore removed on day 5. Serum liver enzyme levels were transiently elevated postoperatively, but rapidly decreased again. There was no rise in total bilirubin or ammonia levels postoperatively. The patient was discharged on postoperative day 8 with no other complications. AST = Aspartate aminotransferase; ALT = alanine aminotransferase; NH3 = ammonia; $\mathrm{T}-\mathrm{Bil}=$ total bilirubin; $\mathrm{POD}=$ postoperative day. 